DESY 05-028

Edinburgh 2005/02

LTH 647

LU-ITP 2005/012

February 2005

\title{
A Determination of the Lambda Parameter from Full Lattice QCD
}

\author{
M. Göckeler ${ }^{1,2}$, R. Horsley ${ }^{3}$, A. C. Irving ${ }^{4}$, D. Pleiter ${ }^{5}$, \\ P. E. L. Rakow ${ }^{4}$, G. Schierholz ${ }^{5,6}$ and H. Stüben ${ }^{7}$ \\ - QCDSF-UKQCD Collaboration - \\ ${ }^{1}$ Institut für Theoretische Physik, Universität Leipzig, \\ D-04109 Leipzig, Germany \\ ${ }^{2}$ Institut für Theoretische Physik, Universität Regensburg, \\ D-93040 Regensburg, Germany \\ 3 School of Physics, University of Edinburgh, \\ Edinburgh EH9 3JZ, UK \\ ${ }^{4}$ Department of Mathematical Sciences, University of Liverpool, \\ Liverpool L69 3BX, UK \\ 5 John von Neumann-Institut für Computing NIC, \\ Deutsches Elektronen-Synchrotron DESY, \\ D-15738 Zeuthen, Germany \\ ${ }^{6}$ Deutsches Elektronen-Synchrotron DESY, \\ D-22603 Hamburg, Germany \\ 7 Konrad-Zuse-Zentrum für Informationstechnik Berlin, \\ D-14195 Berlin, Germany
}

\begin{abstract}
We present a determination of the QCD parameter $\Lambda$ in the quenched approximation $\left(n_{f}=0\right)$ and for two flavours $\left(n_{f}=2\right)$ of light dynamical quarks. The calculations are performed on the lattice using $O(a)$ improved Wilson fermions and include taking the continuum limit. We find $\Lambda_{n_{f}=0}^{\overline{M S}}=$ $259(1)(20) \mathrm{MeV}$ and $\Lambda_{n_{f}=2}^{\overline{M S}}=261(17)(26) \mathrm{MeV}$, using $r_{0}=0.467 \mathrm{fm}$ to set the scale. Extrapolating our results to five flavours, we obtain for the running coupling constant at the mass of the $Z$ boson $\alpha_{s}^{\overline{M S}}\left(m_{Z}\right)=$ $0.112(1)(2)$.
\end{abstract}




\section{Introduction}

The parameter $\Lambda$ is one of the fundamental quantities of QCD. It sets the scale for the running coupling constant $\alpha_{s}(\mu)$, and it is the only parameter of the theory in the chiral limit. Usually $\Lambda$ is defined by writing $\alpha_{s}(\mu)$ as an expansion in inverse powers of $\ln \left(\mu^{2} / \Lambda^{2}\right)$. For such a relationship to remain valid for all values of $\mu$, $\Lambda$ must change as flavour thresholds are crossed: $\Lambda \rightarrow \Lambda_{n_{f}}$, where $n_{f}$ indicates the effective number of light (with respect to the scale $\mu$ ) quarks.

A lattice calculation of $\Lambda$ requires an accurate determination of a reference scale, the introduction of an appropriate non-perturbatively defined coupling, which can be computed accurately on the lattice over a sufficiently wide range of energies, as well as a reliable extrapolation to the chiral and continuum limits. Finally, and equally importantly, one needs to know the relation of the coupling to

$\alpha_{s}^{\overline{M S}}$, the quantity of final interest, accurately to a few percent. This programme has been achieved for the pure gauge theory [1, 2]. In full QCD calculations with Wilson fermions the amount of lattice data was barely enough to enable a reliable chiral and continuum extrapolation [2, 3]. Recent calculations with staggered fermions cover a wider range of lattice spacings and quark masses [4]. However, staggered fermions are not without their own problems.

We determine $\Lambda$ in the $\overline{M S}$ scheme from the force parameter $r_{0}[5]$ and the 'boosted' coupling $g_{\square}$. The latter is obtained from the average plaquette. The advantage of this method is that both quantities are known to high precision. As in our previous work 2, 3, we shall use here non-perturbatively $O(a)$ improved Wilson (clover) fermions. Definitions of the action are standard (see, for example, Appendix D of [6]). The lattice calculations will be done for $n_{f}=2$ flavours of dynamical quarks. In addition, we will update our quenched results.

Since our first attempt [2, 3] the amount of lattice data with dynamical quarks has greatly increased [7]. That is to say, at our previous couplings $\beta=5.20$, 5.25 and 5.29 we have increased the statistics and done additional simulations at smaller quark masses. Furthermore, we have generated dynamical gauge field configurations at $\beta=5.40$ for three different quark masses. At each $\beta$ value we now have data at three to four quark masses at our disposal, and the smallest lattice spacing that we have reached in our simulations is $a \approx 0.07 \mathrm{fm}$. This allows us to improve on, and disentangle, the chiral and continuum extrapolations. In the quenched case the force parameter $r_{0} / a$ is now known up to $\beta=6.92$ [] .

The paper is organised as follows. In section 2 we present a general discussion about the $\beta$ function, including Padé approximations, and the running coupling constant. Also given are results in the $\overline{M S}$ scheme. In section 3 we set up the lattice formalism and discuss what coefficients are known. Various possibilities for converting to the $\overline{M S}$ scheme are given, which will indicate the magnitude of systematic errors. In section 4 results are given for $r_{0} \Lambda^{\overline{M S}}$ for both quenched $\left(n_{f}=0\right)$ and unquenched $n_{f}=2$ fermions. These results are then extrapolated 
to $n_{f}=3$ flavours of dynamical quarks in section [5. This is done by matching the static force at the scale $r_{0}$. In section [6 we convert our results to physical units and, after matching $\alpha_{s}$ to $n_{f}=5$ flavours, compare them with other lattice determinations and to the experimental values. Finally, in section 7 we give our conclusions.

\section{The QCD Coupling and the $\beta$ Function}

The 'running' of the QCD coupling constant as the scale changes is controlled by the $\beta$ function,

$$
\frac{\partial g_{\mathcal{S}}(M)}{\partial \log M}=\beta^{\mathcal{S}} g_{\mathcal{S}}(M)
$$

with

$$
\beta^{\mathcal{S}}\left(g_{\mathcal{S}}\right)=-b_{0} g_{\mathcal{S}}^{3}-b_{1} g_{\mathcal{S}}^{5}-b_{2}^{\mathcal{S}} g_{\mathcal{S}}^{7}-b_{3}^{\mathcal{S}} g_{\mathcal{S}}^{9}-\ldots,
$$

renormalisation having introduced a scale $M$ together with a scheme $\mathcal{S}$. The first two coefficients are scheme independent and are given for the $S U(3)$ colour gauge group as

$$
b_{0}=\frac{1}{(4 \pi)^{2}}\left(11-\frac{2}{3} n_{f}\right), \quad b_{1}=\frac{1}{(4 \pi)^{4}}\left(102-\frac{38}{3} n_{f}\right) .
$$

Integrating eq. (11) gives

$$
\frac{\Lambda^{\mathcal{S}}}{M}=F^{\mathcal{S}}\left(g_{\mathcal{S}}(M)\right)
$$

with

$$
F^{\mathcal{S}}\left(g_{\mathcal{S}}\right)=\exp \left(-\frac{1}{2 b_{0} g_{\mathcal{S}}^{2}}\right)\left(b_{0} g_{\mathcal{S}}^{2}\right)^{-\frac{b_{1}}{2 b_{0}^{2}}} \exp \left\{-\int_{0}^{g_{\mathcal{S}}} d \xi\left[\frac{1}{\beta^{\mathcal{S}}(\xi)}+\frac{1}{b_{0} \xi^{3}}-\frac{b_{1}}{b_{0}^{2} \xi}\right]\right\}
$$

where $\Lambda^{\mathcal{S}}$, the integration constant, is the fundamental scheme dependent QCD parameter. The integral in eq. (5) may be performed numerically or to low orders analytically. For example, to 3 loops we have

$$
\frac{\Lambda^{\mathcal{S}}}{M}=\exp \left(-\frac{1}{2 b_{0} g_{\mathcal{S}}^{2}}\right)\left(b_{0} g_{\mathcal{S}}^{2}\right)^{-\frac{b_{1}}{2 b_{0}^{2}}}\left(1+\frac{A^{\mathcal{S}}}{2 b_{0}} g_{\mathcal{S}}^{2}\right)^{-p_{A}^{\mathcal{S}}}\left(1+\frac{B^{\mathcal{S}}}{2 b_{0}} g_{\mathcal{S}}^{2}\right)^{-p_{B}^{\mathcal{S}}}
$$

where

$$
\begin{aligned}
& A^{\mathcal{S}}=b_{1}+\sqrt{b_{1}^{2}-4 b_{0} b_{2}^{\mathcal{S}}}, \\
& B^{\mathcal{S}}=b_{1}-\sqrt{b_{1}^{2}-4 b_{0} b_{2}^{\mathcal{S}}},
\end{aligned}
$$




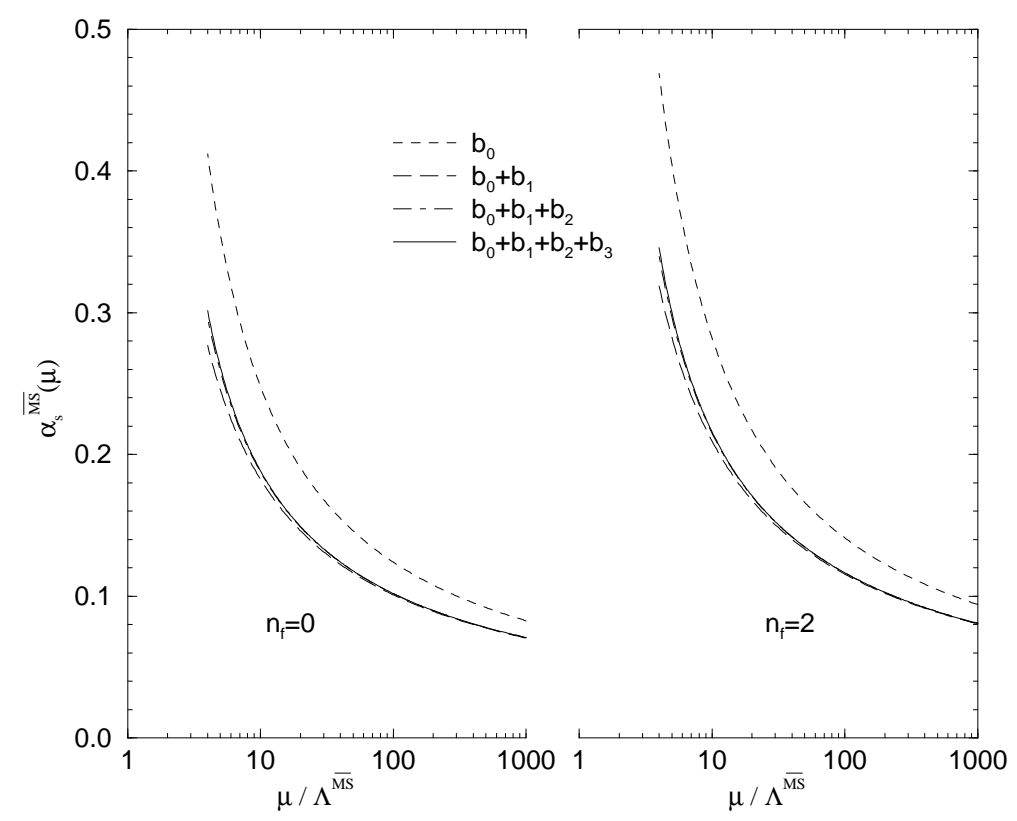

Figure 1: $\alpha_{s}^{\overline{M S}}(\mu)$ versus $\mu / \Lambda^{\overline{M S}}$ for $n_{f}=0$ (left picture) and $n_{f}=2$ (right picture), using successively more and more coefficients of the $\beta$ function.

and

$$
\begin{aligned}
& p_{A}^{\mathcal{S}}=-\frac{b_{1}}{4 b_{0}^{2}}-\frac{b_{1}^{2}-2 b_{0} b_{2}^{\mathcal{S}}}{4 b_{0}^{2} \sqrt{b_{1}^{2}-4 b_{0} b_{2}^{\mathcal{S}}}}, \\
& p_{B}^{\mathcal{S}}=-\frac{b_{1}}{4 b_{0}^{2}}+\frac{b_{1}^{2}-2 b_{0} b_{2}^{\mathcal{S}}}{4 b_{0}^{2} \sqrt{b_{1}^{2}-4 b_{0} b_{2}^{\mathcal{S}}}} .
\end{aligned}
$$

Results are usually given in the $\overline{M S}$ scheme, with the scale $M$ being replaced by $\mu$, and thus

$$
\frac{\Lambda^{\overline{M S}}}{\mu}=F^{\overline{M S}}\left(g_{\overline{M S}}(\mu)\right) .
$$

In this scheme the next two $\beta$ function coefficients are known [9, 10, 11]:

$$
\begin{aligned}
b_{2}^{\overline{M S}}=\frac{1}{(4 \pi)^{6}}\left(\frac{2857}{2}-\right. & \left.\frac{5033}{18} n_{f}+\frac{325}{54} n_{f}^{2}\right) \\
b_{3}^{\overline{M S}}=\frac{1}{(4 \pi)^{8}}\left[\frac{149753}{6}+\right. & 3564 \zeta_{3}-\left(\frac{1078361}{162}+\frac{6508}{27} \zeta_{3}\right) n_{f} \\
& \left.+\left(\frac{50065}{162}+\frac{6472}{81} \zeta_{3}\right) n_{f}^{2}+\frac{1093}{729} n_{f}^{3}\right] .
\end{aligned}
$$

The running coupling $\alpha_{s}^{\overline{M S}}(\mu) \equiv g_{\overline{M S}}^{2}(\mu) / 4 \pi$ is plotted in Fig. 1 for $n_{f}=0,2$ by solving eq. (51) numerically, using only the first coefficient (1-loop), the first and 
second coefficients (2-loop) etc. of the $\beta$ function. The figure shows an apparently rapidly convergent series (cf the 3- to 4-loop result), certainly in the range we will be interested in, $\mu / \Lambda^{\overline{M S}} \sim 20$. The main difference between the $n_{f}=0$ and $n_{f}=2$ results is that $\left.\alpha_{s}^{\overline{M S}}\right|_{n_{f}=2}$ rises more steeply as a function of $\mu / \Lambda^{\overline{M S}}$, as $\left.b_{0}\right|_{n_{f}=2}<\left.b_{0}\right|_{n_{f}=0}$.

A knowledge of the $\beta$ function to 4 loops is the exception rather than the rule. In many schemes it is known only to 3 loops. To improve the convergence of the $\beta$ function, we may attempt to use a Padé approximation by writing eq. (2) as

$$
\beta_{[1 / 1]}^{\mathcal{S}}\left(g_{\mathcal{S}}\right)=-\frac{b_{0} g_{\mathcal{S}}^{3}+\left(b_{1}-\frac{b_{0} b_{2}^{\mathcal{S}}}{b_{1}}\right) g_{\mathcal{S}}^{5}}{1-\frac{b_{2}^{\mathcal{S}}}{b_{1}} g_{\mathcal{S}}^{2}},
$$

which on expanding is arranged to give the first three coefficients of eq. (21) and estimates the next coefficient $b_{3}^{\mathcal{S}}$ as

$$
b_{3}^{\mathcal{S}} \approx \frac{\left(b_{2}^{\mathcal{S}}\right)^{2}}{b_{1}} .
$$

It is again possible to give an analytic result for $F^{\mathcal{S}}$ using $\beta_{[1 / 1]}^{\mathcal{S}}$. We find

$$
\frac{\Lambda^{\mathcal{S}}}{M}=\exp \left(-\frac{1}{2 b_{0} g_{\mathcal{S}}^{2}}\right)\left[\frac{b_{0} g_{\mathcal{S}}^{2}}{1+\left(\frac{b_{1}}{b_{0}}-\frac{b_{2}^{\mathcal{S}}}{b_{1}}\right) g_{\mathcal{S}}^{2}}\right]^{-\frac{b_{1}}{2 b_{0}^{2}}} .
$$

At least for the $\overline{M S}$ scheme this appears to work reasonably well. Equation (12) gives $b_{3}^{\overline{M S}} \approx 3.22 \times 10^{-5}$ and $1.67 \times 10^{-5}$ for quenched and unquenched fermions, respectively, to be compared with the true values from eq. (10) of $4.70 \times 10^{-5}$ and $2.73 \times 10^{-5}$. In 3 we have shown a figure of the various Padé approximations to the $\beta$ function. In Fig. 2 we show the value of $F^{\overline{M S}}\left(g_{\overline{M S}}\right)$ at $g_{\overline{M S}}^{2}=2$ versus the $\beta$ function coefficient number for both quenched and unquenched fermions. Also shown are the results using the $[1 / 1]$ Padé approximations. It is seen that these numbers lie extremely close to the 4-loop $\beta$ function results. As Padé approximations give some estimation of the effect of higher order $\beta$ function coefficients, we shall thus prefer these later in our determination of the $\Lambda$ parameter.

\section{Lattice Methods}

On the lattice we also have a coupling constant $g_{0}(a)$ and corresponding $\beta$ function with coefficients $b_{i}^{L A T}$ and parameter $\Lambda^{L A T}$, where

$$
a \Lambda^{L A T}=F^{L A T}\left(g_{0}(a)\right) .
$$




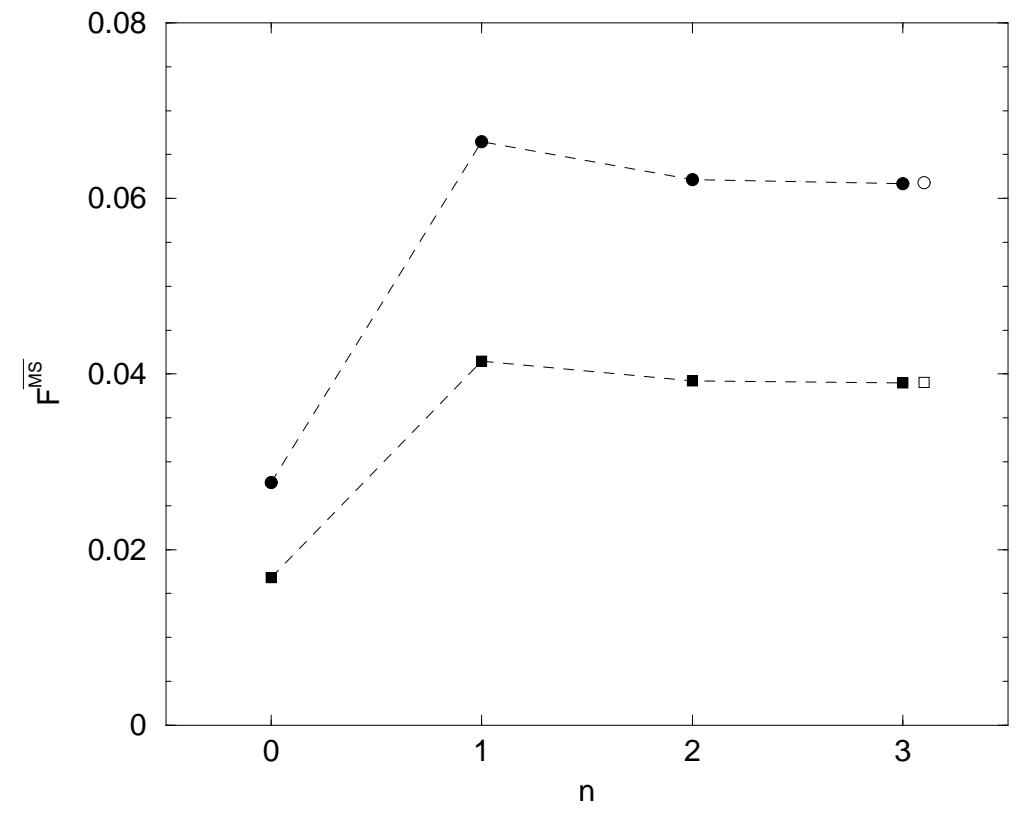

Figure 2: $F^{\overline{M S}}\left(g_{\overline{M S}}\right)$ for $g_{\overline{M S}}^{2}=2$ versus $\beta$ function coefficient number $n$. The $n_{f}=0$ values are filled circles, while the $n_{f}=2$ values are filled squares. The [1/1] Padé approximations are given as open symbols.

To evaluate $F^{L A T}$, we need to know the $b_{i}^{L A T}$ s. They can be found by expanding $g_{\overline{M S}}$ as a power series in $g_{0}$ as

$$
\begin{aligned}
\frac{1}{g_{\overline{M S}}^{2}(\mu)} & =\frac{1}{g_{0}^{2}(a)}+2 b_{0} \ln a \mu-t_{1}^{L A T}+\left(2 b_{1} \ln a \mu-t_{2}^{L A T}\right) g_{0}^{2}(a) \\
& +\left[-2 b_{0} b_{1} \ln ^{2} a \mu+2\left(b_{2}^{\overline{M S}}+b_{1} t_{1}^{L A T}\right) \ln a \mu-t_{3}^{L A T}\right] g_{0}^{4}(a)+\ldots
\end{aligned}
$$

To have consistency between eqs. (9) and (14) we need

$$
t_{1}^{L A T}=2 b_{0} \ln \frac{\Lambda^{\overline{M S}}}{\Lambda^{L A T}},
$$

and

$$
\begin{aligned}
& b_{2}^{L A T}=b_{2}^{\overline{M S}}+b_{1} t_{1}^{L A T}-b_{0} t_{2}^{L A T}, \\
& b_{3}^{L A T}=b_{3}^{\overline{M S}}+2 b_{2}^{\overline{M S}} t_{1}^{L A T}+b_{1}\left(t_{1}^{L A T}\right)^{2}-2 b_{0} t_{3}^{L A T},
\end{aligned}
$$

where $b_{i}^{L A T}$ are the lattice $\beta$ function coefficients, as in eq. (21). So the transformation between the two schemes is given by the $t_{i}^{L A T}$ (which define the transformation), and the renormalisation group dictates how the scale running occurs (in this case the $\ln a \mu$ terms). A knowledge of (the 1-loop) $t_{1}^{L A T}$ determines the relationship between the $\Lambda$ parameters in the two schemes, while also knowing (the 2-loop) $t_{2}^{L A T}$ means that the 3-loop $\beta$ function coefficient $b_{2}^{L A T}$ can be found. 
At present, what we know is 12, 13, 14, 15, 16, 17, 2]

$$
\begin{aligned}
t_{1}^{L A T} & =0.4682013-n_{f}\left[0.0066960-0.0050467 c_{s w}+0.0298435 c_{s w}^{2}\right. \\
& \left.+a m_{q}\left(-0.0272837+0.0223503 c_{s w}-0.0070667 c_{s w}^{2}\right)+O\left(\left(a m_{q}\right)\right)^{2}\right], \\
t_{2}^{L A T} & =0.0556675-n_{f}\left[0.002600+0.000155 c_{s w}-0.012834 c_{s w}^{2}\right. \\
& \left.-0.000474 c_{s w}^{3}-0.000104 c_{s w}^{4}+O\left(a m_{q}\right)\right] .
\end{aligned}
$$

Here $t_{1}^{L A T}$ has been calculated including the $a m_{q}$ terms $\left(m_{q}\right.$ being the bare quark mass), while $t_{2}^{L A T}$ is known only for $a m_{q}=0$, and $t_{3}^{L A T}$ is unknown, which means that from eq. (17) $b_{2}^{L A T}$ is known but not $b_{3}^{L A T}$. For general $c_{s w}$ the connection between $g_{\overline{M S}}^{2}$ and $g_{0}^{2}$ is only defined up to terms of $O(a)$, but on the improvement trajectory $c_{s w}=1+O\left(g_{0}^{2}\right)$ it is possible to arrange it to be $O\left(a^{2}\right)$ if the $a m_{q}$ terms are included in the $t_{i}^{L A T} \mathrm{~s}$.

Thus, the conversion from the lattice coupling to the $\overline{M S}$ coupling (eqs. (15) and (18) ) can also be written with mass independent $t_{i}^{L A T} \mathrm{~s}$, if we redefine $g_{0}^{2}$ by replacing it by $\tilde{g}_{0}^{2}$, where

$$
\tilde{g}_{0}^{2}=g_{0}^{2}\left(1+b_{g} a m_{q}\right), \quad b_{g}=b_{g}^{(0)} n_{f} g_{0}^{2}+O\left(g_{0}^{4}\right) .
$$

So, putting $c_{s w}=1+O\left(g_{0}^{2}\right)$ into eqs. (15) and (18) means that $t_{1}^{L A T}$ is replaced by $t_{1}^{L A T}-n_{f} a m_{q} b_{g}^{(0)}$, which gives $b_{g}^{(0)}=0.01200$. This value agrees with the number reported in [18].

Thus, in this mass independent scheme (ie a scheme where the renormalisation conditions are imposed for zero quark mass) there appears to be little difference in extrapolating to the chiral limit using constant $\beta=6 / g_{0}^{2}$, rather than constant $\tilde{\beta}=6 / \tilde{g}_{0}{ }^{2}$. So, rather than using eq. (18) at finite $a m_{q}$, we shall first extrapolate our plaquette and $r_{0} / a$ data to the chiral limit and then determine $\Lambda^{\overline{M S}}$. Before attempting this, we shall discuss some improvements to help improve the convergence of the power series (15).

As it is well known that lattice perturbative expansions are poorly convergent, we have used a 'boosted' coupling constant

$$
g_{\square}^{2} \equiv \frac{g_{0}^{2}(a)}{u_{0}^{4}}
$$

to help the series (15), or equivalently (2) for $\beta^{L A T}\left(g_{0}\right)$, converge faster. Here $P \equiv u_{0}^{4}=\left\langle\operatorname{Tr} U^{\square}\right\rangle / 3$ is the average plaquette. In perturbation theory we write

$$
\frac{1}{g_{\square}^{2}}=\frac{1}{g_{0}^{2}}-p_{1}-p_{2} g_{0}^{2}+O\left(g_{0}^{4}\right)
$$

with [19, 20]

$$
\begin{aligned}
& p_{1}=\frac{1}{3}, \\
& p_{2}=0.0339110-n_{f}\left(0.001846-0.0000539 c_{s w}+0.001590 c_{s w}^{2}\right)
\end{aligned}
$$


for massless clover fermions.

To improve the convergence of the series further, we re-express it in terms of the tadpole improved coefficient

$$
c_{s w}^{\square}=c_{s w} u_{0}^{3} .
$$

Changing $t_{i}^{L A T}$ to $t_{i}^{\square}$ first replaces $t_{i}^{L A T}$ by $t_{i}^{L A T}-p_{i}$, and secondly using $c_{s w}^{\square}$ simply replaces every $c_{s w}$ by $c_{s w}^{\square}$ in $t_{1}^{L A T}$, but the change in $t_{2}^{\square}$ is more complicated as the coefficients of $c_{s w}^{\square}$ change in $t_{2}^{\square}$.

This gives for $t_{i}^{\square} \equiv t_{i}^{\square}\left(c_{s w}^{\square}\right)$ in the chiral limit

$$
\begin{aligned}
t_{1}^{\square} & =0.1348680-n_{f}\left[0.0066960-0.0050467 c_{s w}^{\square}+0.0298435\left(c_{s w}^{\square}\right)^{2}\right] \\
t_{2}^{\square} & =0.0217565-n_{f}\left[0.000753+0.001053 c_{s w}^{\square}-0.000498\left(c_{s w}^{\square}\right)^{2}\right. \\
& \left.-0.000474\left(c_{s w}^{\square}\right)^{3}-0.000104\left(c_{s w}^{\square}\right)^{4}\right] .
\end{aligned}
$$

As we have here a 2-loop result, we can see how well tadpole improvement improves the series convergence. The coefficient of $n_{f}$ in $t_{2}^{\square}$ is considerably smaller than the corresponding coefficient in $t_{2}^{L A T}$. For example, using the values at $\beta=5.40$ given in the next section, we find that the magnitude of the coefficient is reduced by two orders of magnitude (from $\sim-0.0438$ to $\sim 0.0003$ ).

What this tadpole improvement represents is taking a path from $g^{2}=0$ to $g^{2}=g_{\square}^{2}$, keeping $c_{s w}^{\square}$ fixed. Later we shall consider other trajectories from 0 to $g_{\square}^{2}$. If we had all orders of the theory, the result would depend only on the end point. But with a finite series the trajectory will matter. This will help us estimate systematic errors from unknown higher order terms.

Thus, in conclusion we have

$$
\begin{aligned}
a \Lambda^{\square} & =F^{\square}\left(g_{\square}(a)\right), \\
\frac{\Lambda^{\overline{M S}}}{\mu} & =F^{\overline{M S}}\left(g_{\overline{M S}}(\mu)\right),
\end{aligned}
$$

together with the conversion formula

$$
\frac{1}{g_{\overline{M S}}^{2}(\mu)}=\frac{1}{g_{\square}^{2}(a)}+2 b_{0} \ln a \mu-t_{1}^{\square}+\left(2 b_{1} \ln a \mu-t_{2}^{\square}\right) g_{\square}^{2}(a)+\ldots
$$

with

$$
t_{1}^{\square}=2 b_{0} \ln \frac{\Lambda^{\overline{M S}}}{\Lambda^{\square}}
$$

and

$$
b_{2}^{\square}=b_{2}^{\overline{M S}}+b_{1} t_{1}^{\square}-b_{0} t_{2}^{\square} .
$$

We shall now discuss various strategies to determine $\Lambda^{\overline{M S}}$. 


\subsection{Method I}

This method was used in our previous papers [2, 3, 21, with the difference that now we first extrapolate to the chiral limit. For each $\beta$ value we first compute $t_{i}^{\square}$ from eq. (24). Then from eq. (27) we convert $g_{\square}$ to $g_{\overline{M S}}$ at some appropriate scale $\mu_{*}$, and using the force scale $r_{0}$, we calculate $r_{0} \Lambda^{\overline{M S}}$ from eq. (26):

$$
r_{0} \Lambda^{\overline{M S}}=r_{0} \mu_{*} F^{\overline{M S}}\left(g_{\overline{M S}}\left(\mu_{*}\right)\right) \text {. }
$$

Finally, we extrapolate to the continuum limit, $a \rightarrow 0$. Note that $t_{i}^{\square}$ will depend on the coupling because $c_{s w}^{\square}$ does.

We must determine the scale $\mu_{*}$. A good choice to help eq. (27) converge rapidly is to take the $O(1)$ coefficient to vanish, which is achieved by choosing [13.

$$
\mu_{*}=\frac{1}{a} \exp \left(\frac{t_{1}^{\square}}{2 b_{0}}\right) .
$$

Thus, we used

$$
\frac{1}{g_{\overline{M S}}^{2}\left(\mu_{*}\right)}=\frac{1}{g_{\square}^{2}(a)}+\left(\frac{b_{1}}{b_{0}} t_{1}^{\square}-t_{2}^{\square}\right) g_{\square}^{2}(a)+O\left(g_{\square}^{4}\right)
$$

to find $g_{\overline{M S}}^{2}\left(\mu_{*}\right)$, which was then substituted into eq. (30).

\subsection{Method II}

Alternatively, we can first determine $b_{2}^{\square}$ from eq. (29) and then determine $r_{0} \Lambda^{\square}$ via eq. (25). After computing this, we convert to $r_{0} \Lambda^{\overline{M S}}$ using

$$
r_{0} \Lambda^{\overline{M S}}=r_{0} \Lambda^{\square} \exp \left(\frac{t_{1}^{\square}}{2 b_{0}}\right)
$$

and then take the continuum limit. Again, note that $b_{2}^{\square}$ will depend on the coupling, because $c_{s w}^{\square}$ does.

This method is equivalent to choosing a scale $\mu_{=}$, as in method I, such that $g_{\overline{M S}}\left(\mu_{=}\right)=g_{\square}(a)$. In this case all the coefficient terms of eq. (27) vanish. The scale that achieves this is

$$
\mu_{=}=\frac{1}{a} \exp \left(\frac{t_{1}^{\square}}{2 b_{0}}\right) \frac{F^{\square}\left(g_{\square}(a)\right)}{F^{\overline{M S}}\left(g_{\square}(a)\right)} .
$$

Indeed, substituting $\mu_{=}$into eq. (26) then gives eq. (33) again. The scale $\mu_{=}$is close to $\mu_{*}$, as can be seen by expanding eq. (34) to 3 loops. From eq. (6) we 
have

$$
\begin{aligned}
\mu_{=} & =\frac{1}{a} \exp \left(\frac{t_{1}^{\square}}{2 b_{0}}\right) \frac{\left(1+\frac{A^{\square}}{2 b_{0}} g_{\square}^{2}\right)^{-p_{A}^{\square}}}{\left(1+\frac{A^{\overline{M S}}}{2 b_{0}} g_{\square}^{2}\right)^{-p_{A}^{\overline{M S}}}} \frac{\left(1+\frac{B^{\square}}{2 b_{0}} g_{\square}^{2}\right)^{-p_{B}^{\square}}}{\left(1+\frac{B^{\overline{M S}}}{2 b_{0}} g_{\square}^{2}\right)^{-p_{B}^{\overline{M S}}}} \\
& =\mu_{*}\left(1-\frac{b_{1} t_{1}^{\square}-b_{0} t_{2}^{\square}}{2 b_{0}^{2}} g_{\square}^{2}+\ldots\right) \\
& >\mu_{*},
\end{aligned}
$$

for the couplings used here.

\subsection{Method III}

Another possibility, and theoretically the most sound, is to vary $c_{s w}^{\square}$ along the improvement path as $g_{\square}^{2}$ increases. This will give genuinely constant $\beta$ function coefficients (ie independent of the coupling). As the 1-loop expansion for $c_{s w}^{\square}$ is known along this path,

$$
c_{s w}^{\square}=1+c_{0}^{\square} g_{\square}^{2}+\ldots,
$$

with $c_{0}^{\square}=c_{0}-\frac{3}{4} p_{1}$ and $c_{0}=0.2659(1)$ [22], then expanding eq. (24) gives

$$
b_{2}^{\square}=b_{2}^{\overline{M S}}+\left.b_{1} t_{1}^{\square}\right|_{c_{s w}^{\square}=1}-\left.b_{0} t_{2}^{\square}\right|_{c_{s w}^{\square}=1}-\left.b_{0} c_{0}^{\square} \frac{\partial t_{1}^{\square}}{\partial c_{s w}^{\square}}\right|_{c_{s w}^{\square}=1}=-0.0008241 .
$$

This result may also be derived from eq. (27) by first setting $a=\mu^{-1}$ (for simplicity) and then taking $\mu \partial / \partial \mu$ of this equation. This leads to

$$
-\frac{2}{g_{\overline{M S}}^{3}} \beta^{\overline{M S}}\left(g_{\overline{M S}}\right)=\left[-\frac{2}{g_{\square}^{3}}-\frac{\partial t_{1}^{\square}}{\partial c_{s w}^{\square}} \frac{\partial c_{s w}^{\square}}{\partial g_{\square}}-2 t_{2}^{\square} g_{\square}+O\left(g_{\square}^{3}\right)\right] \beta^{\square}\left(g_{\square}\right),
$$

which upon expanding out also gives eq. (37).

So, having determined $b_{2}^{\square}$ in eq. (29), the method is as for method II: first determine $r_{0} \Lambda^{\square}$ using eq. (25) and then convert to $r_{0} \Lambda^{\overline{M S}}$ using eq. (33).

\subsection{Methods IIP and IIIP}

To further improve our calculations, and to reduce the systematic error, we consider here the effect of Padé improving the $\beta$ function, as given in eqs. (11) and (13). We restrict ourselves to methods II and III, and we call the Padé improved results IIP and IIIP, respectively. 


\begin{tabular}{||c|c|c|c|c|c||}
\hline \hline$\beta$ & $r_{0} / a$ & $P$ & $r_{0} \Lambda^{\overline{M S}} \mathrm{I}$ & $r_{0} \Lambda^{\overline{M S}} \mathrm{II}$ & $r_{0} \Lambda^{\overline{M S}} \mathrm{IIP}$ \\
\hline 5.70 & $2.922(09)$ & $0.549195(25)$ & $0.4888(15)$ & $0.4950(15)$ & $0.4888(15)$ \\
5.80 & $3.673(05)$ & $0.567651(21)$ & $0.5142(07)$ & $0.5200(07)$ & $0.5140(07)$ \\
5.95 & $4.898(12)$ & $0.588006(20)$ & $0.5461(13)$ & $0.5514(14)$ & $0.5457(13)$ \\
6.00 & $5.368(33)$ & $0.593679(08)$ & $0.5579(34)$ & $0.5631(35)$ & $0.5575(34)$ \\
6.07 & $6.033(17)$ & $0.601099(18)$ & $0.5696(16)$ & $0.5746(16)$ & $0.5692(16)$ \\
6.20 & $7.380(26)$ & $0.613633(02)$ & $0.5861(21)$ & $0.5907(21)$ & $0.5855(21)$ \\
6.40 & $9.740(50)$ & $0.630633(04)$ & $0.5976(31)$ & $0.6018(31)$ & $0.5970(31)$ \\
6.57 & $12.18(10)$ & $0.643524(15)$ & $0.6029(48)$ & $0.6067(48)$ & $0.6022(48)$ \\
6.69 & $14.20(12)$ & $0.651936(15)$ & $0.6055(50)$ & $0.6091(51)$ & $0.6049(50)$ \\
6.81 & $16.54(12)$ & $0.659877(13)$ & $0.6080(46)$ & $0.6113(46)$ & $0.6073(46)$ \\
6.92 & $19.13(15)$ & $0.666721(12)$ & $0.6145(47)$ & $0.6177(47)$ & $0.6139(47)$ \\
\hline$\infty$ & $\infty$ & 1 & $0.6152(21)$ & $0.6189(21)$ & $0.6145(20)$ \\
\hline \hline
\end{tabular}

Table 1: The quenched $r_{0} \Lambda^{\overline{M S}}$ values for methods I, II and IIP (ie using the Padé improved $\beta$ function $\beta_{[1 / 1]}^{\square}$ ) together with the force parameter $r_{0} / a[8]$ (the number at $\beta=6.0$ is from the interpolation formula given there) and the plaquette $P$. The continuum extrapolated values together with the statistical errors are given in the bottom row. Numbers in italics are not used in the fits.

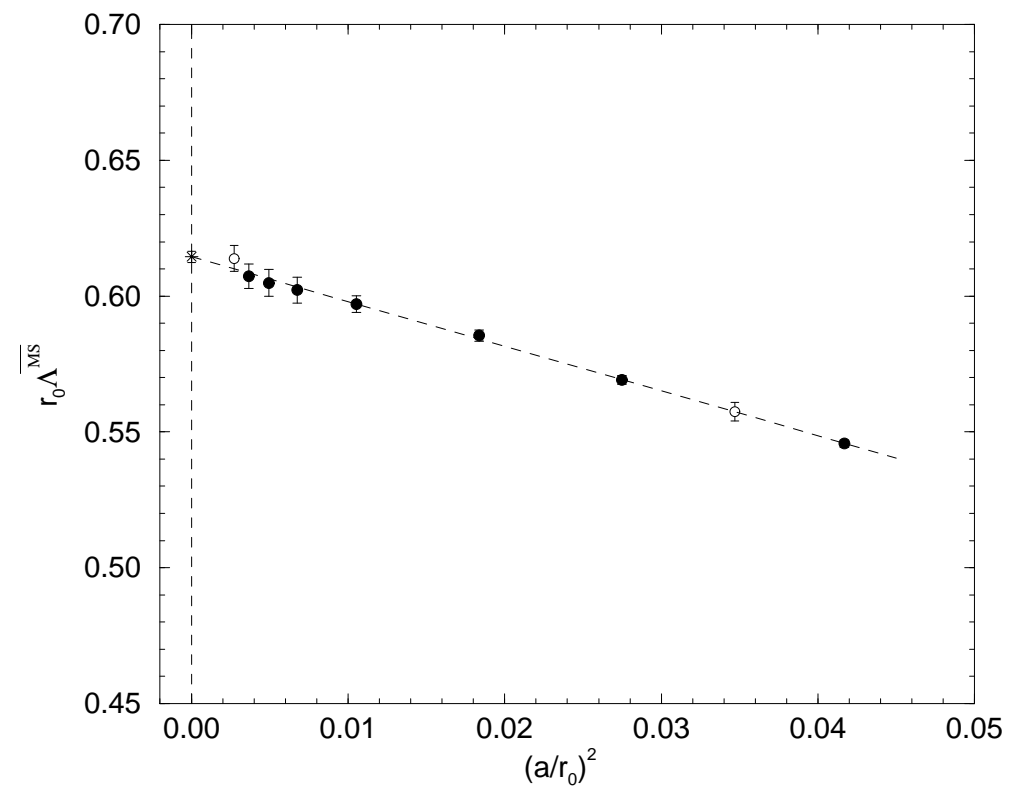

Figure 3: The quenched $r_{0} \Lambda^{\overline{M S}}$ points versus $\left(a / r_{0}\right)^{2}$, together with a linear extrapolation to the continuum limit for method IIP. The filled circles are used for the extrapolation. The star represents the extrapolated value. 


\section{Results}

\subsection{Quenched Results}

In the quenched case $\left(n_{f}=0\right)$ we do not have any of the additional chiral limit extrapolation complications alluded to in the previous section, or a $c_{s w}$ term. This means that there is no difference between method II and method III, so the procedure is straightforward. In Table 1 we give the parameters used. For $r_{0}$ we use, for consistency, exclusively the values given in [8], which includes previous results from [23]. The one exception is $\beta=6.0$, where we have used the interpolation formula [8] for $r_{0} / a$. Our plaquette values are determined at their given $\beta$ values.

In Table 1 we also give the results for $r_{0} \Lambda^{\overline{M S}}$ from methods I, II and IIP. We first see that the results for $r_{0} \Lambda^{\overline{M S}}$ are almost indistinguishable between methods I, II and IIP. Method IIP lies just below method I (and indeed is almost identical with it).

We now consider the continuum limit of our results. In Fig. 3 we plot the results for $r_{0} \Lambda^{\overline{M S}}$ against $\left(a / r_{0}\right)^{2}$ for method IIP. The differences between the results of the various methods are small. As one expects that Padé improvement gives a better answer, we shall concentrate on IIP. The smallest $a$ value is not included in the fit, as it appears to deviate a little, but including it would not have changed the extrapolated value much. We also have not included $\beta=6.0$ in the fit, as $r_{0} / a$ is only known from an interpolation formula. But as can be seen from the figure, including it has no effect on the result. Also, the two coarsest $a$ values have not been included in the fit, as they show significant non-linear effects in $a^{2}$. These two points are not shown in the plot, as they lie far to the right. Figure 3 clearly shows a linear extrapolation over a wide range of lattice spacings, $a^{-1} \sim 2-6.5 \mathrm{GeV}$, giving a value for method IIP of

$$
\left.r_{0} \Lambda_{0}^{\overline{M S}} \equiv r_{0} \Lambda^{\overline{M S}}\right|_{n_{f}=0}=0.614(2)(5)
$$

Here the first error is statistical, and the second systematic error is estimated by the spread in the results between methods I, II and IIP. That the systematic error is small is an indication of the convergence of results from the different methods. The result (39) agrees with our earlier value [2].

\subsection{Unquenched $n_{f}=2$ Results}

We now turn to unquenched $n_{f}=2$ fermions. In Table 2 we show the $\beta, \kappa$ and $c_{s w}$ parameters used in the simulations, together with the measured $r_{0} / a$ and plaquette $P$ values. As discussed in section 3, we shall first determine $r_{0} \Lambda^{\overline{M S}}$ in the chiral limit and then perform the continuum extrapolation. We must thus first find the zero quark mass results from Table 2. We shall make a chiral 


\begin{tabular}{||c|l|c|c|l|l|l||}
\hline \hline$\beta$ & \multicolumn{1}{|c|}{$\kappa$} & $V$ & $c_{s w}$ & \multicolumn{1}{|c|}{$r_{0} / a$} & \multicolumn{1}{c||}{$P$} & Group \\
\hline 5.20 & 0.1342 & $16^{3} \times 32$ & 2.0171 & $4.077(70)$ & $0.528994(58)$ & QCDSF \\
5.20 & 0.1350 & $16^{3} \times 32$ & 2.0171 & $4.754(45)$ & $0.533670(40)$ & UKQCD \\
5.20 & 0.1355 & $16^{3} \times 32$ & 2.0171 & $5.041(53)$ & $0.536250(30)$ & UKQCD \\
5.20 & 0.13565 & $16^{3} \times 32$ & 2.0171 & $5.250(75)$ & $0.537070(100)$ & UKQCD \\
5.20 & 0.1358 & $16^{3} \times 32$ & 2.0171 & $5.320(95)$ & $0.537670(30)$ & UKQCD \\
\hline 5.25 & 0.1346 & $16^{3} \times 32$ & 1.9603 & $4.737(50)$ & $0.538770(41)$ & QCDSF \\
5.25 & 0.1352 & $16^{3} \times 32$ & 1.9603 & $5.138(55)$ & $0.541150(30)$ & UKQCD \\
5.25 & 0.13575 & $24^{3} \times 48$ & 1.9603 & $5.532(40)$ & $0.543135(15)$ & QCDSF \\
\hline 5.29 & 0.1340 & $16^{3} \times 32$ & 1.9192 & $4.813(82)$ & $0.542400(50)$ & UKQCD \\
5.29 & 0.1350 & $16^{3} \times 32$ & 1.9192 & $5.227(75)$ & $0.545520(29)$ & QCDSF \\
5.29 & 0.1355 & $24^{3} \times 48$ & 1.9192 & $5.566(64)$ & $0.547094(23)$ & QCDSF \\
5.29 & 0.1359 & $24^{3} \times 48$ & 1.9192 & $5.880(100)$ & $0.548286(57)$ & QCDSF \\
\hline 5.40 & 0.1350 & $24^{3} \times 48$ & 1.8228 & $6.092(67)$ & $0.559000(19)$ & QCDSF \\
5.40 & 0.1356 & $24^{3} \times 48$ & 1.8228 & $6.381(53)$ & $0.560246(10)$ & QCDSF \\
5.40 & 0.1361 & $24^{3} \times 48$ & 1.8228 & $6.714(64)$ & $0.561281(08)$ & QCDSF \\
\hline \hline
\end{tabular}

Table 2: The unquenched $\beta, \kappa$ and $c_{s w}$ values and the volume $\mathrm{V}$, together with the measured force parameter $r_{0} / a$ and plaquette $P$. The collaboration that generated the configurations is given in the last column. The results for $\beta=5.29$, $\kappa=0.1359$ are preliminary. We have reanalysed our $r_{0} / a$ values, taking autocorrelations properly into account, which gave larger error bars than previously reported [24].

\begin{tabular}{||c|c|c|l||}
\hline \hline$\beta$ & $\kappa_{c}$ & $r_{0} / a$ & \multicolumn{1}{|c||}{$P$} \\
\hline 5.20 & $0.136008(15)$ & $5.455(96)$ & $0.538608(49)$ \\
5.25 & $0.136250(07)$ & $5.885(79)$ & $0.544780(89)$ \\
5.29 & $0.136410(09)$ & $6.254(99)$ & $0.549877(109)$ \\
5.40 & $0.136690(22)$ & $7.390(26)$ & $0.562499(46)$ \\
\hline \hline
\end{tabular}

Table 3: The critical values for $\kappa$ (ie $\kappa_{c}$ ) and the chiral limit values for $r_{0} / a$ and $P$ for the four $\beta$ values used here. 


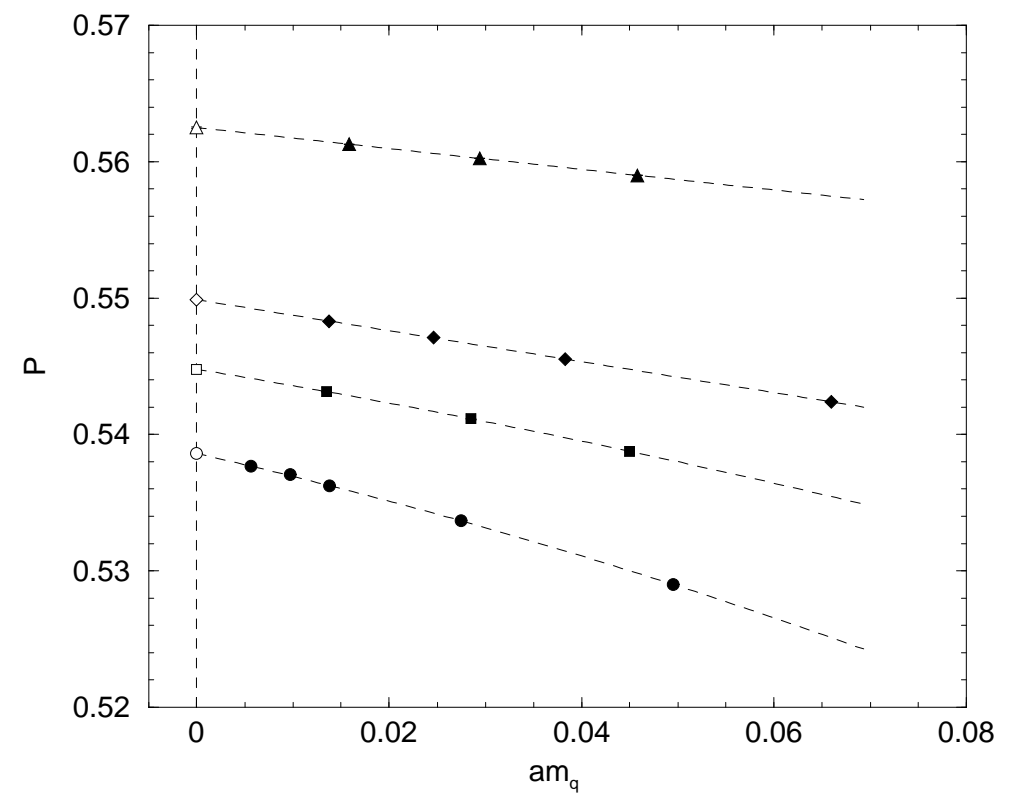

Figure 4: The plaquette $P$ (filled symbols) plotted against the bare quark mass $a m_{q}$ for $\beta=5.20$ (lower curve) until $\beta=5.40$ (upper curve). The fits use eq. (41), giving the extrapolated values in the chiral limit (open symbols).

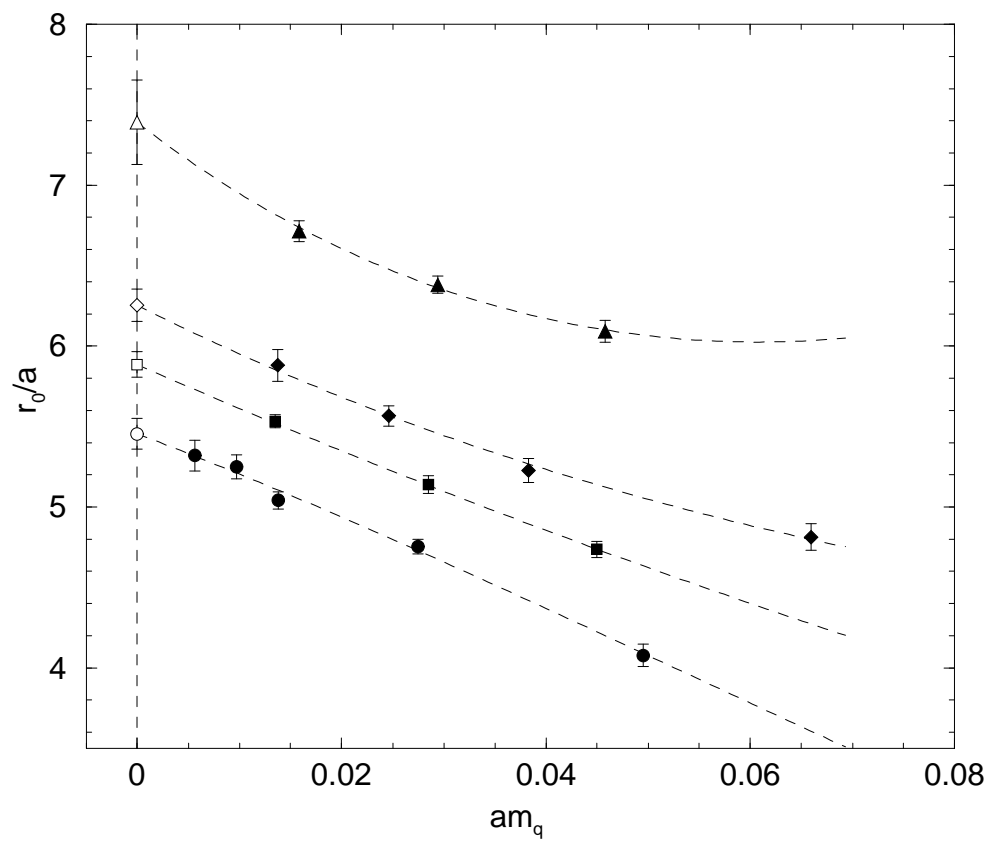

Figure 5: The force parameter $r_{0} / a$ plotted against $a m_{q}$. The same notation as in Fig. 4 is used. 


\begin{tabular}{||c|l|l|l|l|l||}
\hline \hline$\beta$ & $r_{0} \Lambda^{\overline{M S}} \mathrm{I}$ & $r_{0} \Lambda^{\overline{M S}} \mathrm{II}$ & $r_{0} \Lambda^{\overline{M S}} \mathrm{IIP}$ & $r_{0} \Lambda^{\overline{M S}} \mathrm{III}$ & $r_{0} \Lambda^{\overline{M S}} \mathrm{IIIP}$ \\
\hline 5.20 & $0.5183(91)$ & $0.5304(94)$ & $0.4913(87)$ & $0.6459(114)$ & $0.6173(109)$ \\
5.25 & $0.5210(71)$ & $0.5415(73)$ & $0.5040(68)$ & $0.6450(87)$ & $0.6174(83)$ \\
5.29 & $0.5372(85)$ & $0.5482(87)$ & $0.5120(81)$ & $0.6433(102)$ & $0.6165(98)$ \\
5.40 & $0.5577(198)$ & $0.5676(201)$ & $0.5343(189)$ & $0.6431(228)$ & $0.6182(219)$ \\
\hline$\infty$ & $0.6012(346)$ & $0.6085(352)$ & $0.5819(329)$ & $0.6376(412)$ & $0.6170(395)$ \\
\hline \hline
\end{tabular}

Table 4: The values for $r_{0} \Lambda^{\overline{M S}}$ for methods I, II, IIP, III, IIIP described in section 3 for the four $\beta$ values used here.

extrapolation in $a m_{q}$, defined here by

$$
a m_{q}=\frac{1}{2}\left(\frac{1}{\kappa}-\frac{1}{\kappa_{c}}\right) .
$$

We estimate $\kappa_{c}$ from partially quenched pion data. The results have been given in [7] and are tabulated in the second column of Table 3,

In Fig. 4 we show the results for the plaquette. For each $\beta$ value the data appear to be rather linear in the quark mass $a m_{q}$, in particularly for the higher $\beta$ values. This suggests that a quadratic fit ansatz is sufficient to obtain the value of $P$ in the chiral limit. We thus use

$$
P=d_{0}+d_{1} a m_{q}+d_{2}\left(a m_{q}\right)^{2} .
$$

Except for $\beta=5.20$, it does not much matter whether a linear or quadratic fit is used. In Fig. 5 we show the results for $r_{0} / a$. The data is less linear in $a m_{q}$, and also less smooth, so we used the renormalisation group inspired global fit ansatz

$$
\ln \frac{r_{0}}{a}=A_{1}(\beta)+A_{2}(\beta) a m_{q}+A_{3}(\beta)\left(a m_{q}\right)^{2},
$$

where $A_{1}(\beta)$ is a linear polynomial in $\beta$, and $A_{2}(\beta), A_{3}(\beta)$ are quadratic polynomials in $\beta$. This ansatz was also used in [7. The results of the fits in the chiral limit are given in Table 3 ,

In Table 4 we give our results for $r_{0} \Lambda^{\overline{M S}}$ for methods I, II, IIP, III and IIIP. Again, as the results for method I are very similar to method II, we shall not discuss method I further here. In Fig. 6 we plot $r_{0} \Lambda^{\overline{M S}}$ against $\left(a / r_{0}\right)^{2}$ for methods IIP and IIIP, together with a linear extrapolation to the continuum limit. Though we cannot reach such small $a$ values as for the quenched case, the $r_{0} \Lambda^{\overline{M S}}$ data do seem to lie on straight lines. We find a linear behaviour at least over the region $a^{-1} \sim 2-3 \mathrm{GeV}$. This seems to be well inside the linear region of Fig. 3 ,

For methods IIP (and II) the results lie roughly parallel to the quenched results, while for methods IIIP (and III) they are flatter and higher. However, in the continuum limit they agree within error bars. Ideally, the result should 


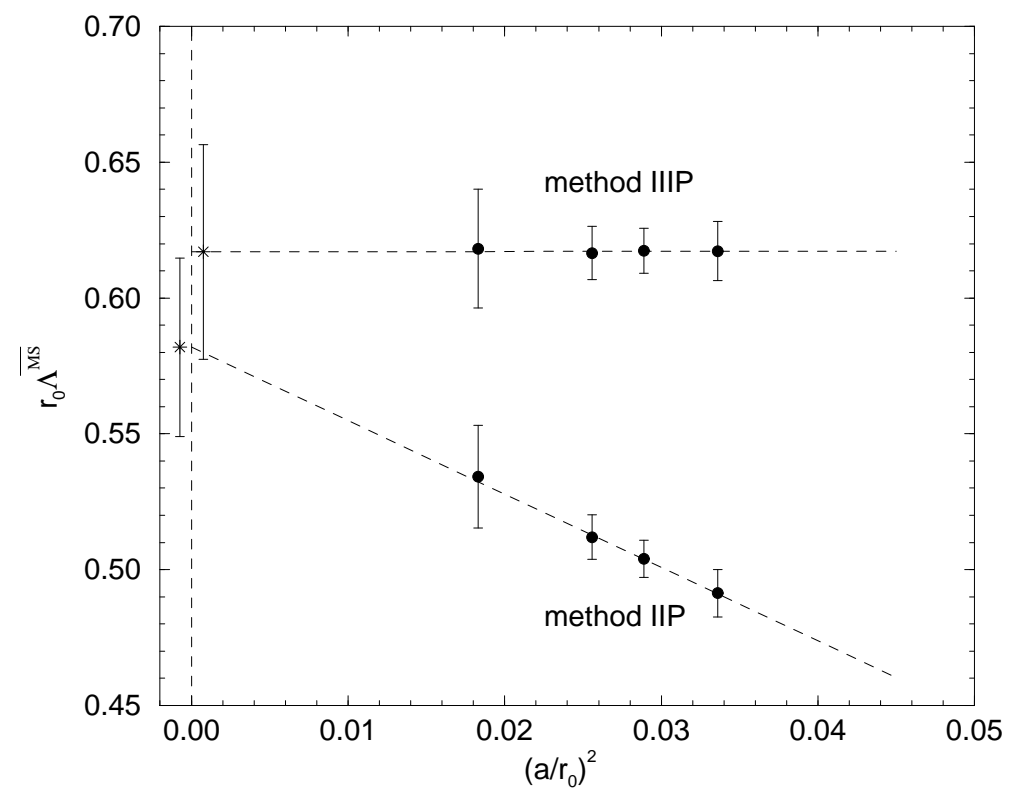

Figure 6: The unquenched $r_{0} \Lambda^{\overline{M S}}$ points (filled circles) versus $\left(a / r_{0}\right)^{2}$, together with a linear extrapolation to the continuum limit for methods IIP and IIIP. Stars represent the extrapolated values.

not depend on the choice of trajectory. The way this should work, as mentioned before, is that although the coefficients $t_{i}^{\square}$ will be different depending on the path one might choose, the sum

$$
\frac{1}{g_{\square}^{2}(a)}-t_{1}^{\square}-t_{2}^{\square} g_{\square}^{2}(a)+\ldots
$$

should not. However, at the order to which we have the series this is not yet so. The difference between methods II and III is that we have replaced $c_{s w}^{\square}$ by its 1-loop expansion. Returning to Fig. 6, the fact that the results from methods II, IIP are almost parallel to the quenched results suggests that in methods II, IIP the $O\left(a^{2}\right)$ effects come from the same source as in the quenched case, which must be the gluon action. For methods III, IIIP the slope is much smaller so there must have been a fortuitous cancellation between $a^{2}$ effects from the gluon and fermion terms.

One expects that Padé improvement gives a better answer, so the $\mathrm{P}$ results are more trustworthy. Previous experience suggests that the procedure in IIP of using tadpole improved $c_{s w}$ works fairly well. For example, $\kappa_{c}$ in [25] and the renormalisation constant $Z$ for $v_{2 b}$ in [6] agree within a few percent with the non-perturbative values. However, method IIIP is a more consistent approach. Furthermore, the results from method IIIP appear to be insensitive to the particular form of the continuum extrapolation. We therefore take these numbers as 
our best estimate.

From the linear extrapolation of method IIIP to the continuum limit we thus quote

$$
\left.r_{0} \Lambda_{2}^{\overline{M S}} \equiv r_{0} \Lambda^{\overline{M S}}\right|_{n_{f}=2}=0.617(40)(21),
$$

where the first error is statistical and the second systematic. The latter error is estimated by the spread in the results between method III and IIIP. Compared to our previous result [2], the value (44) has increased by $\approx 10 \%$, but still lies within the error bars.

\section{$5 \quad$ Extrapolation to $n_{f}=3$ Flavours}

At high energy scales we can see that $\Lambda^{\overline{M S}}$ makes some fairly large jumps as we pass through the heavy quark mass thresholds and change the effective number of flavours. From [26] we can see that the reason for these large jumps is the fact that $m_{q} / \Lambda^{\overline{M S}}$ is large. We want to argue here that the situation with light quarks, $m_{q} \lesssim \Lambda^{\overline{M S}}$, is rather different, and that in this case we do not expect to see any dramatic dependence of $\Lambda^{\overline{M S}}$ on $n_{f}$.

We will determine the $n_{f}=3$ flavour $\Lambda$ parameter from matching the static force at the scale $r_{0}$.

\subsection{One-loop Matching}

To make clear what is involved in matching, we will go through the 1-loop calculation in some detail.

At the 1-loop level the static potential between fundamental charges is given by

$$
\begin{aligned}
V(r)=-\frac{4}{3} \frac{g_{\overline{M S}}^{2}(\mu)}{4 \pi r}\left\{1+\frac{g_{\overline{M S}}^{2}(\mu)}{16 \pi^{2}}[\right. & 22\left(\ln \mu r+\gamma_{E}+\frac{31}{66}\right) \\
& \left.\left.-\frac{4}{3} n_{f}\left(\ln \mu r+\gamma_{E}+\frac{5}{6}\right)\right]+\cdots\right\}
\end{aligned}
$$

for massless sea quarks (see, for example, [27]). We can work out the force $f(r)$ at distance $r$ by differentiating this to give

$$
\begin{aligned}
4 \pi r^{2} f(r)=\frac{4}{3} g_{\overline{M S}}^{2}(\mu)\left\{1+\frac{g_{\overline{M S}}^{2}(\mu)}{16 \pi^{2}}[\right. & 22\left(\ln \mu r+\gamma_{E}-\frac{35}{66}\right) \\
& \left.\left.-\frac{4}{3} n_{f}\left(\ln \mu r+\gamma_{E}-\frac{1}{6}\right)\right]+\cdots\right\} .
\end{aligned}
$$


If we now change the flavour number from 2 to 0 , or from 2 to 3 , while keeping the force at distance $r$ constant, we get

$$
\begin{array}{r}
33 \ln \frac{\Lambda_{0}^{\overline{M S}}}{\Lambda_{2}^{\overline{M S}}}=-4\left(\ln \Lambda_{2}^{\overline{M S}} r+\gamma_{E}-\frac{1}{6}\right), \\
(33-6) \ln \frac{\Lambda_{3}^{\overline{M S}}}{\Lambda_{2}^{\overline{M S}}}=2\left(\ln \Lambda_{2}^{\overline{M S}} r+\gamma_{E}-\frac{1}{6}\right) .
\end{array}
$$

We can eliminate $r$ from these equations, leaving us with the simple equation

$$
\frac{\Lambda_{3}^{\overline{M S}}}{\Lambda_{2}^{\overline{M S}}}=\left(\frac{\Lambda_{2}^{\overline{M S}}}{\Lambda_{0}^{\overline{M S}}}\right)^{\frac{11}{18}}
$$

which can be used to estimate $\Lambda_{3}^{\overline{M S}}$ from the $n_{f}=0$ and $n_{f}=2$ results.

\section{$5.2 \quad$ Higher Loops}

To repeat this matching calculation with more loops, we follow [8] and define a force-scale coupling $g_{q \bar{q}}$ by

$$
4 \pi r^{2} f(r) \equiv \frac{4}{3} g_{q \bar{q}}^{2}(r)
$$

From eq. (46) we can read off

$$
t_{1}^{q \bar{q}}=-\frac{1}{(4 \pi)^{2}}\left[22\left(\gamma_{E}-\frac{35}{66}\right)-\frac{4}{3} n_{f}\left(\gamma_{E}-\frac{1}{6}\right)\right] .
$$

We can find $t_{2}^{q \bar{q}}$ by calculating the force from the 2-loop expression of $V(r)$ reported in [28, 29]:

$$
\begin{aligned}
t_{2}^{q \bar{q}}=\frac{1}{(4 \pi)^{4}}[ & \frac{1107}{2}-204 \gamma_{E}-\frac{229}{3} \pi^{2}+\frac{9}{4} \pi^{4}-66 \zeta_{3} \\
& \left.+\frac{n_{f}}{3}\left(-\frac{553}{3}+76 \gamma_{E}+\frac{44}{3} \pi^{2}+52 \zeta_{3}\right)+\frac{4}{27} n_{f}^{2}\left(12-\pi^{2}\right)\right]
\end{aligned}
$$

which gives us enough information to calculate the 3-loop $\beta$ function for $g_{q \bar{q}}(r)$ (cf eq. (17)). There would be complications in going to the next order, because it is known that terms of the type $\alpha_{s}^{4} \ln \alpha_{s}$ will enter the series for the potential [30].

We are now ready to see how $\Lambda^{\overline{M S}}$ depends on flavour number, if we make the value of $f(r)$ independent of $n_{f}$ (the number of massless quark flavours) at some particular $r$ value. Implicitly, we assume $r \approx r_{0}$. If $f(r)$ is independent of $n_{f}$, 


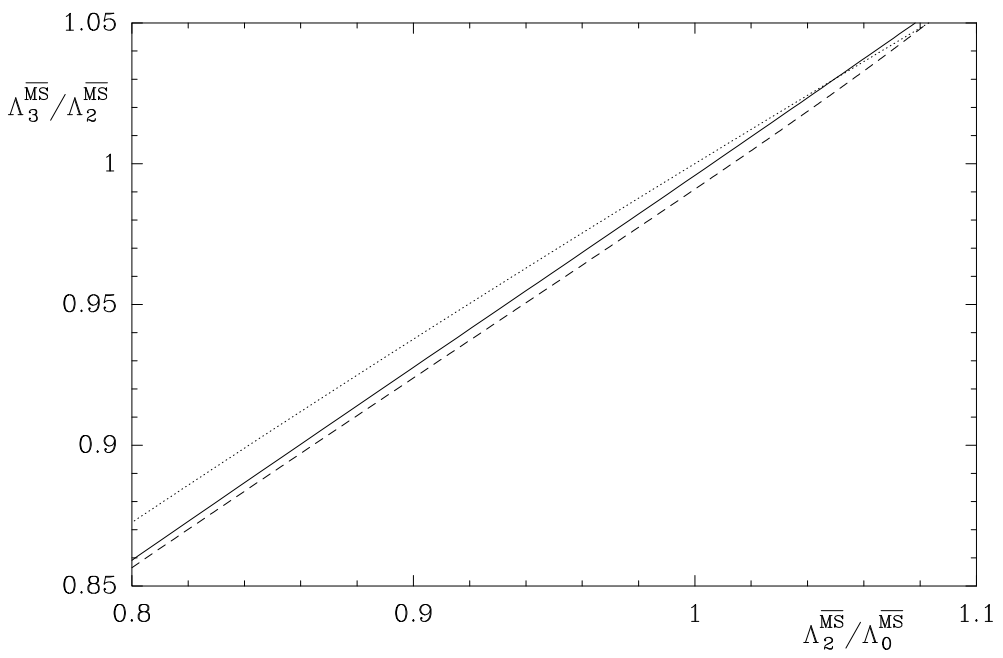

Figure 7: The ratio $\Lambda_{3}^{\overline{M S}} / \Lambda_{2}^{\overline{M S}}$ against the ratio $\Lambda_{2}^{\overline{M S}} / \Lambda_{0}^{\overline{M S}}$ from 1-loop (dotted line), 2-loop (dashed line) and 3-loop (solid line) matching.

then $g_{q \bar{q}}(r)$ is independent of $n_{f}$ too. We can compare the $q \bar{q}$ scheme $\Lambda$ s by using

$$
\begin{aligned}
& r \Lambda_{0}^{q \bar{q}}=F^{q \bar{q}}\left(g_{q \bar{q}}(r), n_{f}=0\right), \\
& r \Lambda_{2}^{q \bar{q}}=F^{q \bar{q}}\left(g_{q \bar{q}}(r), n_{f}=2\right), \\
& r \Lambda_{3}^{q \bar{q}}=F^{q \bar{q}}\left(g_{q \bar{q}}(r), n_{f}=3\right) .
\end{aligned}
$$

We can take ratios of these equations to cancel $r$ and find equations for $\Lambda$ ratios. These $q \bar{q}$ scheme $\Lambda$ ratios can then be converted into $\overline{M S}$ by using $t_{1}^{q \bar{q}}$ from eq. (50). This gives us a way of making a parametric plot of $\Lambda$ ratios by varying $g_{q \bar{q}}$ and calculating all three $\Lambda$ s from $g_{q \bar{q}}$. In Fig. [7] we show the plot.

The results clearly have to be treated with some caution, because $r_{0} \Lambda$ is a fairly large number. So it is not clear how much we can learn from perturbative results at the scale $r_{0}$. It is therefore quite surprising that the different orders of perturbation theory agree so well in Fig. [7. Furthermore, we have assumed in this section that $r_{0} m_{s} \ll 1$, so that the strange quark can reasonably be treated as massless. Both these difficulties could be decreased by using a smaller distance (and thus a smaller value for $r^{2} f(r)$ ) to set our scale.

\subsection{Result for $n_{f}=3$}

From our quenched and unquenched $n_{f}=2$ results, (39) and (44), we obtain $\Lambda_{2}^{\overline{M S}} / \Lambda_{0}^{\overline{M S}}=1.005$. If we insert this number into the 3-loop matching curve shown in Fig. [7, we find $\Lambda_{3}^{\overline{M S}} / \Lambda_{2}^{\overline{M S}}=0.999$. From this ratio and eq. (44) we then 
obtain for $n_{f}=3$ quark flavours

$$
\left.r_{0} \Lambda_{3}^{\overline{M S}} \equiv r_{0} \Lambda^{\overline{M S}}\right|_{n_{f}=3}=0.616(29)(19)
$$

We have not attempted to estimate the systematic error induced by the matching procedure.

\section{Comparison with Phenomenology}

In this section we shall make a comparison with other lattice and phenomenological results. For this we first need to set the force scale in terms of a physical unit.

To fix the scale $r_{0}$ in physical units, we extrapolate recent dimensionless nucleon masses $m_{N} r_{0}$ found by the CP-PACS, JLQCD and QCDSF-UKQCD collaborations jointly to the physical pion mass following [24]. This gives the value $r_{0}=0.467 \mathrm{fm}$ with an estimated error of $7 \%$. We will use this number throughout this paper. A similar result for $r_{0}$ was quoted in 31.

For the quenched case we then obtain

$$
\Lambda_{0}^{\overline{M S}}=259(1)(20) \mathrm{MeV}
$$

and for the unquenched case we find

$$
\begin{aligned}
& \Lambda_{2}^{\overline{M S}}=261(17)(26) \mathrm{MeV} \\
& \Lambda_{3}^{\overline{M S}}=260(12)(26) \mathrm{MeV}
\end{aligned}
$$

The systematic errors quoted here include the uncertainty in setting the scale. Note that previously [2] we had assumed $r_{0}=0.5 \mathrm{fm}$.

In Fig. 8 we show our results for $\Lambda^{\overline{M S}}$ together with recent experimental values from 32 and 33 . It appears that the lattice results extrapolate smoothly to the experimental values at $n_{f}=4\left[32\right.$ and $n_{f}=5$ [33]. However, our $n_{f}=3$ result lies two standard deviations below the corresponding phenomenological value (open triangle). (The reader should be aware that the sometimes called experimental numbers imply a good deal of modelling and, thus, should be regarded as phenomenological numbers.)

In order to compare $\alpha_{s}$ from various experiments and theory, it must be evolved to a common scale. For convenience this is taken to be the mass of the $Z$ boson, $m_{Z}$. Having computed $\Lambda^{\overline{M S}}$ for $n_{f}=3$ flavours, we may use the 4-loop expansion of $\alpha_{s}$ and the 3-loop matching condition at the quark thresholds [26, 34]

to determine $\alpha_{n_{f}=5}^{\overline{M S}}\left(m_{Z}\right)$. We take the charm and bottom thresholds to be at 1.5 and $4.5 \mathrm{GeV}$, respectively. Furthermore, we choose the charm and bottom quark masses to be $m_{c}^{\overline{M S}}\left(m_{c}\right)=1.5 \mathrm{GeV}$ and $m_{b}^{\overline{M S}}\left(m_{b}\right)=4.5 \mathrm{GeV}$, respectively. Varying 


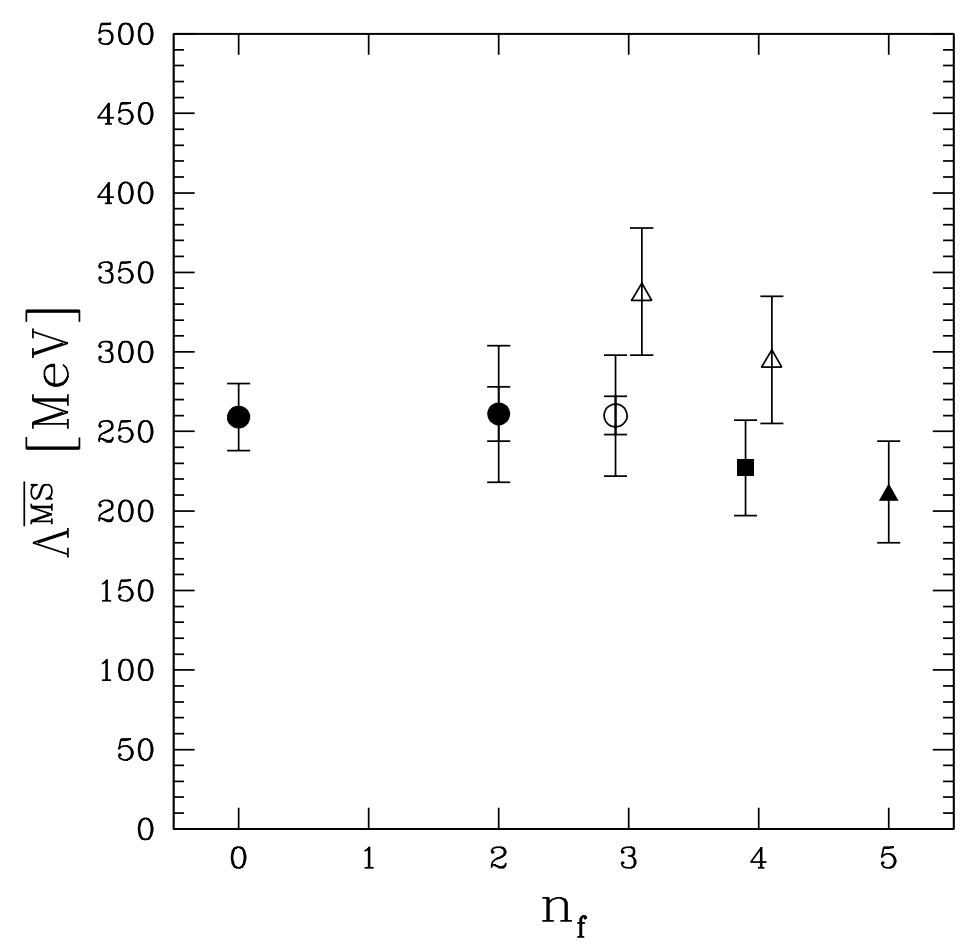

Figure 8: Values of $\Lambda^{\overline{M S}}$ versus number of quark flavours $n_{f}$. The filled circles are our $n_{f}=0,2$ results, and the open circle is our extrapolated value. The inner error bars give the statistical errors, while the outer error bars give the total errors. The square is from a 3-loop analysis of the non-singlet structure functions [32]. The triangles are taken from [33. The open triangles are evaluated using the 4-loop expansion of $\alpha_{s}$ and 3-loop matching at the quark thresholds. The entries at $n_{f}=3$ and 4 have been displaced horizontally.

the charm and bottom quark masses within reasonable limits has a neglible effect on the final result. We then obtain

$$
\alpha_{n_{f}=5}^{\overline{M S}}\left(m_{Z}\right)=0.112(1)(2) .
$$

This is to be compared with the world average value [33] $\alpha_{s}^{\overline{M S}}\left(m_{Z}\right)=0.1182(27)$.

In Fig. 9 we compare our result for $\alpha_{s}^{\overline{M S}}\left(m_{Z}\right)$ with other lattice results and experiment. We find agreement with previous lattice calculations using Wilson fermions. It occurs that the Wilson results lie systematically below the mean experimental value. On the other hand, calculations using staggered fermions (albeit from the same group) show a better agreement with experiment. Our result for $r_{0} \Lambda_{2}^{\overline{M S}}$ agrees also with that of the ALPHA collaboration [40, which does not quote a number for $\alpha_{s}^{\overline{M S}}\left(m_{Z}\right)$. Our result for $\alpha_{s}^{\overline{M S}}\left(m_{Z}\right)$ lies two standard deviations below the phenomenological value. 


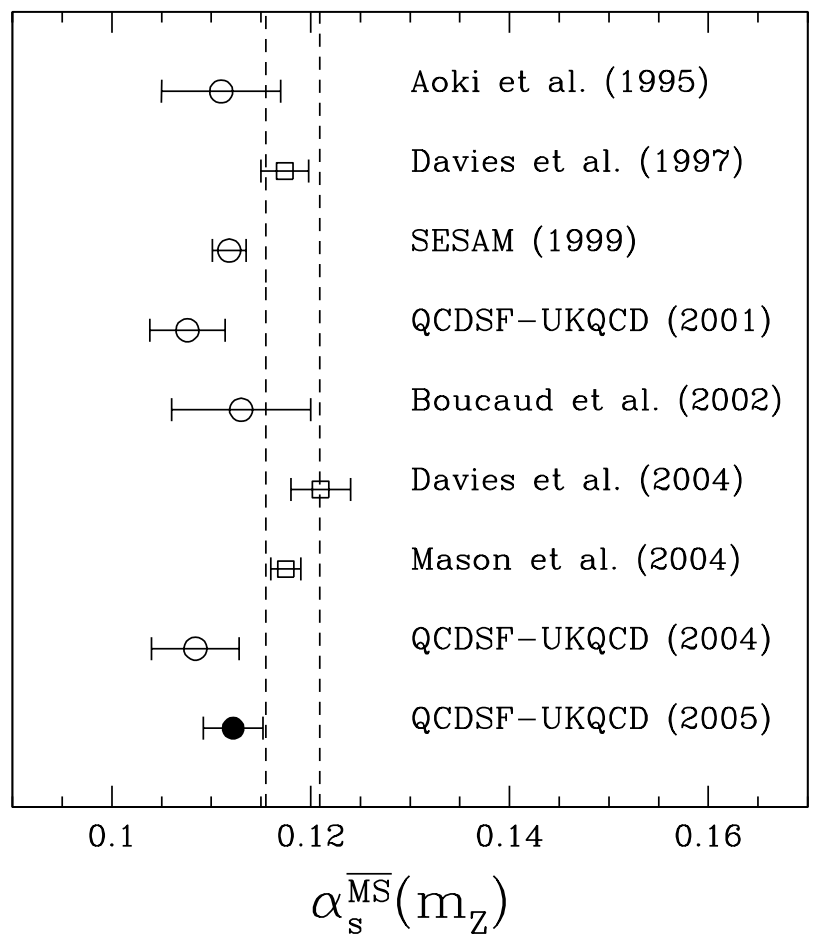

Figure 9: Comparison of $\alpha_{s}^{\overline{M S}}\left(m_{Z}\right)$ from this work (solid circle) with other lattice results 35, 36, 37, 2, 38, 4, 39, 21] (from top to bottom). The circles are from Wilson fermions and the squares from staggered fermions. The dashed line indicates the mean experimental value [33].

\section{Conclusions}

Due to substantial improvements of the performance of our hybrid Monte Carlo algorithm [41, we were able to extend our dynamical simulations to smaller quark masses and to larger values of $\beta$. Our smallest lattice spacing now is $a \approx 0.07 \mathrm{fm}$. This enabled us to perform a chiral and continuum extrapolation of the lattice data. Because the calculation involves a perturbative conversion from the lattice coupling constant to the (mass independent) $\overline{M S}$ constant, it was important to first extrapolate the lattice data to the chiral limit. We have discussed basically two approaches of converting the lattice coupling constant to the $\overline{M S}$ one. They differed mainly in how the non-perturbative improvement (clover) term was incorporated in the perturbative expansion. It was reassuring to see that both methods led to the same result in the continuum limit. This indicates once more that a reliable extrapolation to the continuum limit is very important.

We could also improve on our quenched result, because data at smaller lattice 
spacings became available.

There are several sources of systematic error in our calculation. The main error comes from setting the scale, followed by the continuum extrapolation. As better dynamical data become available, the uncertainty in setting the scale will be gradually reduced. Simulations at smaller lattice spacings will become possible with the next generation of computers, which should facilitate the extrapolation to the continuum limit.

\section{Acknowledgements}

We like to thank Antonios Athenodorou and Haris Panagopoulos for checking the numbers in eq. (22). The numerical calculations have been performed on the Hitachi SR8000 at LRZ (Munich), on the Cray T3E at EPCC (Edinburgh) 42, on the Cray T3E at NIC (Jülich) and ZIB (Berlin), as well as on the APE1000 and Quadrics at DESY (Zeuthen). We thank all institutions. This work has been supported in part by the EU Integrated Infrastructure Initiative Hadron Physics (I3HP) under contract RII3-CT-2004-506078 and by the DFG under contract FOR 465 (Forschergruppe Gitter-Hadronen-Phänomenologie).

\section{References}

[1] S. Capitani, M. Lüscher, R. Sommer and H. Wittig, Nucl. Phys. B544 (1999) 669.

[2] S. Booth, M. Göckeler, R. Horsley, A. C. Irving, B. Joó, S. Pickles, D. Pleiter, P. E. L. Rakow, G. Schierholz, Z. Sroczynski and H. Stüben, Phys. Lett. $\underline{519}$ (2001) 229.

[3] S. Booth, M. Göckeler, R. Horsley, A. C. Irving, B. Joó, S. Pickles, D. Pleiter, P. E. L. Rakow, G. Schierholz, Z. Sroczynski and H. Stüben, Nucl. Phys. Proc. Suppl. B106 (2002) 308.

[4] C. T. H. Davies, E. Follana, A. Gray, G. P. Lepage, Q. Mason, M. Nobes, J. Shigemitsu, H. D. Trottier, M. Wingate, C. Aubin, C. Bernard, T. Burch, C. DeTar, S. Gottlieb, E. B. Gregory, U. M. Heller, J. E. Hetrick, J. Osborn, R. Sugar, D. Toussaint, M. Di Pierro, A. El-Khadra, A. S. Kronfeld, P. B. Mackenzie, D. Menscher and J. Simone, Phys. Rev. Lett. $\underline{92}$ (2004) 022001.

[5] R. Sommer, Nucl. Phys. B411 (1994) 839.

[6] M. Göckeler, R. Horsley, D. Pleiter, P. E. L. Rakow and G. Schierholz, hep-ph/0410187 
[7] M. Göckeler, R. Horsley, A. C. Irving, D. Pleiter, P. E. L. Rakow, G. Schierholz and H. Stüben, hep-ph/0409312.

[8] S. Necco and R. Sommer, Nucl. Phys. B622 (2002) 328.

[9] O. V. Tarasov, A. A. Vladimirov and A. Yu. Zharkov, Phys. Lett. B93 (1980) 429.

[10] S. A. Larin and J. A. M. Vermaseren, Phys. Lett. B303 (1993) 334.

[11] T. van Ritbergen, J. A. M. Vermaseren and S. A. Larin, Phys. Lett. B400 (1997) 379 .

[12] M. Lüscher and P. Weisz, Phys. Lett. B349 (1995) 165.

[13] M. Lüscher, hep-lat/9802029.

[14] B. Allés, A. Feo and H. Panagopoulos, Phys. Lett. B426 (1998) 361.

[15] S. Sint, private notes (1996), quoted in A. Bode, P. Weisz and U. Wolff, Nucl. Phys. $\underline{\mathrm{B} 576}$ (2000) 517.

[16] L. Marcantonio, P. Boyle, C. T. H. Davies, J. Hein and J. Shigemitsu, Nucl. Phys. Proc. Suppl. $\underline{94}$ (2001) 363.

[17] A. Bode and H. Panagopoulos, Nucl. Phys. B625 (2002) 198.

[18] M. Lüscher, S. Sint, R. Sommer and P. Weisz, Nucl. Phys. B478 (1996) 365.

[19] G. S. Bali and P. Boyle, hep-lat/0210033.

[20] A. Athenodorou, H. Panagopoulos and A. Tsapalis, hep-lat/0409127.

[21] M. Göckeler, R. Horsley, A. C. Irving, D. Pleiter, P. E. L. Rakow, G. Schierholz and H. Stüben, hep-lat/0409166.

[22] M. Lüscher and P. Weisz, Nucl. Phys. B479 (1996) 429.

[23] M. Guagnelli, R. Sommer and H. Wittig, Nucl. Phys. B535 (1998) 389.

[24] A. Ali Khan, T. Bakeyev, M. Göckeler, T. R. Hemmert, R. Horsley, A. C. Irving, B. Joó, D. Pleiter, P. E. L. Rakow, G. Schierholz and H. Stüben, Nucl. Phys. B689 (2004) 175.

[25] M. Göckeler, R. Horsley, H. Perlt, P. Rakow, G. Schierholz, A. Schiller and P. Stephenson, Phys. Rev. D57 (1998) 5562.

[26] K. G. Chetyrkin, B. A. Kniehl and M. Steinhauser, Phys. Rev. Lett. $\underline{79}$ (1997) 2184. 
[27] I. Montvay and G. Münster, Quantum Fields on a Lattice, Cambridge University Press (Cambridge, 1994).

[28] Y. Schröder, Phys. Lett. B447 (1999) 321.

[29] M. Peter, Nucl. Phys. B501 (1997) 471.

[30] T. Appelquist, M. Dine and I. J. Muzinich, Phys. Rev. D17 (1978) 2074.

[31] C. Aubin, C. Bernard, C. DeTar, Steven A. Gottlieb, E. B. Gregory, U. M. Heller, J. E. Hetrick, J. Osborn, R. Sugar and D. Toussaint, Phys. Rev. D70 (2004) 094505.

[32] J. Blümlein, H. Böttcher and A. Guffanti, Nucl. Phys. Proc. Suppl. 135 (2004) 152.

[33] S. Bethke, Nucl. Phys. Proc. Suppl. 121 (2003) 74; ibid. 135 (2004) 345.

[34] K. G. Chetyrkin, B. A. Kniehl and M. Steinhauser, Nucl. Phys. B510 (1998) 61; K. G. Chetyrkin, J. H. Kühn and M. Steinhauser, Comp. Phys. Commun. $\underline{133}(2000) 43$.

[35] S. Aoki, M. Fukugita, S. Hashimoto, N. Ishizuka, H. Mino, M. Okawa, T. Onogi and A. Ukawa, Phys. Rev. Lett. $\underline{74}$ (1995) 22.

[36] C. T. H. Davies, K. Hornbostel, G. P. Lepage, P. McCallum, J. Shigemitsu and J. Sloan, Phys. Rev. D56 (1997) 2755.

[37] A. Spitz, H. Hoeber, N. Eicker, S. Güsken, Th. Lippert, K. Schilling, T. Struckmann, P. Ueberholz and J. Viehoff, Phys. Rev. D60 (1999) 074502.

[38] P. Boucaud, J. P. Leroy, H. Moutarde, J. Micheli, O. Pene, J. RodriguezQuintero and C. Roiesnel, JHEP 0201 (2002) 046.

[39] Q. Mason et al., talk at Lattice 2004, and paper to appear.

[40] M. Della Morte, R. Frezzotti, J. Heitger, J. Rolf, R. Sommer and U. Wolff, hep-lat/0411025.

[41] T. Bakeyev, M. Göckeler, R. Horsley, D. Pleiter, P. E. L. Rakow, G. Schierholz and H. Stüben, Phys. Lett. B580 (2004) 197.

[42] C. R. Allton, S. P. Booth, K. C. Bowler, J. Garden, A. Hart, D. Hepburn, A. C. Irving, B. Joó, R. D. Kenway, C. M. Maynard, C. McNeile, C. Michael, S. M. Pickles, J. C. Sexton, K. J. Sharkey, Z. Sroczynski, M. Talevi, M. Teper and H. Wittig, Phys. Rev. D65 (2002) 054502. 